\title{
RCBM: a rough content-based image quality assessment metric
}

\author{
Qian $\mathrm{Yu}^{*}$ \\ Department of Computer Science, \\ University of Regina, \\ Regina, SK, S4S 0A2, Canada \\ E-mail: yu209@cs.uregina.ca \\ *Corresponding author
}

\section{Wei Dong}

Texas Instruments,

12500, TI Blvd., Dallas, Texas, 75243, USA

E-mail: weidong@ti.com

\author{
Chang N. Zhang \\ Department of Computer Science, \\ University of Regina, \\ Regina, SK, S4S 0A2, Canada \\ E-mail: zhang@cs.uregina.ca
}

\begin{abstract}
A generalised content-based image quality assessment technique is proposed in this paper. Different from many existing image quality metrics, where the digital image quality is evaluated by comparing with a reference image and a single 'exact' value is provided for the purpose of 'accurately' quantifying the image quality, our proposed method defines the image quality metric based on the theory of the rough fuzzy integral and a region (with a pair of the boundary values) is presented to estimate the image quality instead of a scalar value. The new philosophy for the proposed 'rough' content-based image quality metric lays on the acceptance of the uncertainty of subjective image quality assessment through the human visual system (HVS) and addresses this kind of uncertainty by applying the rigorous mathematical concept of the rough and fuzzy set on the standard content-based image quality analysis. Therefore, the proposed method is a good mimicking of the subjective image quality assessment and meets the accuracy requirement under the uncertainty measurement of HVS.
\end{abstract}

Keywords: rough sets; fuzzy measure; rough fuzzy integral; image quality assessment; structural similarity.

Reference to this paper should be made as follows: Yu, Q., Dong, W. and Zhang, C.N. (2013) 'RCBM: a rough content-based image quality assessment metric', Int. J. Granular Computing, Rough Sets and Intelligent Systems, Vol. 3, No. 1, pp.44-58. 
Biographical notes: Qian $\mathrm{Yu}$ is a PhD student in the Department of Computer Science at the University of Regina. He received his MSc in Computer Science from the University of Regina in 2007, and BEng in Computer Science and Technology from National University of Defense Technology in 2003.

Wei Dong is currently working at Texas Instruments as a Senior R\&D Engineer. He received his $\mathrm{PhD}$ in Electrical and Computer Engineering from Texas A\&M University, MS in Electrical Engineering from Shanghai Jiao Tong University and BS in Electrical Engineering from Xi'an Jiaotong University, respectively.

Chang N. Zhang has been at the University of Regina since 1990 where he is currently a Professor of the Department of Computer Science, and Adjunct Scientist with Telecommunication Research Labs (TRLabs). He received his BS in Applied Math from the Shanghai University, Shanghai, China, and PhD in Computer Science and Engineering from Southern Methodist University, Dallas, TX, USA.

\section{Introduction}

Image quality assessment is essential to any image processing. A basic quality assessment for an image is developed by comparing the image with the reference so that the judgement can be done to decide how about the quality of the image. A number of the different image quality assessment techniques have been suggested based on the various criteria and employed to the diverse applications.

Among all different image quality assessments, it seems that the most 'correct' evaluation for an image is still through the subjective assessment. This is due to the fact that it is meaningful only if the image is visualised through the human visual system (HVS) and the final purpose of the image processing is for the perception by human beings. However, the subjective assessment usually resorts to a large amount of subjective view experiments. In order to obtain the reliable ratings for the quality of the digital images via the mean opinion scores (MOSs), which requires the abundance of subjective individual samples. Therefore, from the practical engineering point of view, the subject image quality assessment is vulnerable by the external environment and individual factors and difficult to describe by means of the precise mathematical model.

To address the difficulty of the subjective image quality assessment, various objective image quality assessment techniques are employed to accurately and automatically provide quantitative measures that can predict perceived image quality without the subjective individual involved (Eckert and Bradley, 1998; Gao et al., 2005; Mannos and Sakrison, 1974; Pappas et al., 2005; Wang et al., 2004). From the most widely-used error-sensitive metric, such as mean squared error (MSE), and the related quantity of peak signal to noise ratio (PSNR), to the sophisticated HVS model and the knowledge-based method, no matter which kind of the objective image quality assessment method, the goal is always to predict the certain image quality features from the subjective assessment. In other words, the objective image quality assessment is imitative of the subjective image quality assessment to some extent. Therefore, there exists a trade-off between the accuracy and the complexity for objective image quality 
assessment methods. For example, the MSE metric is simply to compute the MSEs of the pixels of the testing image compared with the reference image in the form of

$$
M S E=\frac{1}{N} \sum_{i=1}^{N}\left(x_{i}-y_{i}\right)^{2},
$$

where $N$ is the total number of pixels in the image and $x_{i}$ and $y_{i}$ are the intensities of the $i$-th pixels of the reference and testing images, respectively. And the relevant PSNR is represented as

$$
P S N R=10 \log _{10} \frac{L^{2}}{M S E}
$$

where $L$ is the dynamic range of the allowable pixel intensities. Although, the MSE (or PSNR) metric is relatively simple, it is sometimes problematic as demonstrated in Wang and Bovik (2009). One problem is that the smaller MSE values may not necessarily guarantee the higher perceptual quality. In terms of the conclusion in Miyahara et al. (1998), the 'amount of error', the 'location of error' and the 'structure of error' are three essential factors of distortion. This implies that only the amount of the error itself is unable to always represent the perceptual quality of the testing image.

Facing this kind of the limitation, another type of the content-based objective image quality assessment techniques has been proposed. For this type of the assessment methods, the information and the knowledge of natural visual environment in the entire visual observation process has been used. As a distinct example, a new full-reference image quality assessment scheme is proposed based on the measurement of the structure information change (or structure similarity) (Wang et al., 2004). According to the philosophy of the structural-similarity-based image quality assessment, the natural image signals are highly 'structured' and the signal samples have strong dependencies among themselves. Therefore, the quality of the image can be evaluated by calculating the structural distortion in the image considering that the procedure of visual observation is to extract such kind of structural information. Compared with the simple MSE metric, it may have a better image quality assessment because the 'structure of error' and 'location of error' are also involved to some extent.

Although, in principle, it has been demonstrated that the distortion of the structural information plays an important role to assess the image quality in Wang et al. (2004), how to obtain the structural information has not been completely addressed. The concept of structural similarity (SSIM) index has been employed to quantify the structural distortion for each pixels of the image and the mean structural similarity (MSSIM) index is used as a single overall quality measure of the image in Wang et al. (2004). Furthermore, the authors in Gao et al. (2005) suggested a content-based image quality metric (CBM). This CBM method fuses the amount and local information into the similarity of the image structural information based on fuzzy integral and gives a more comprehensive evaluation for the quality of the specified image.

However, both the MSSIM and the CBM are the pixel-wised assessment metrics. This means that the assessment has to be done by calculating the SSIM index per pixel. Considering the procedure of the CBM method, the correlation and redundancy remain in the clustered pixels in neighbourhood. This indicates that the overall effect of a cluster of pixels in neighbourhood is more important than the value of each pixel. Therefore, it is natural for us to design a metric to reflect the characteristics above. Therefore, a general 
block-wised metric is proposed in this paper. From the experience of the visual observation, it is known that there exists uncertainty for the subjective assessment of the image quality due to the diversity of the individuals and the variations of the observation environment. To reflect the unavoidable uncertainty of the subjective image quality assessment, we introduce the concept of the rough set into the image assessment and propose a rough content-based metric (RCBM) by inducting the rough fuzzy integral to fuse the block-wised local information and providing a pair of the boundary values (lower and upper boundary values) to quantify the image quality. Because the new approach presents a region instead of a value (such as CBM and MSSIM) to define the image quality, although it seems less precise than the exact value, it is more reasonable to assess the image quality to corresponding to the uncertainty of MOS.

The rest of this paper is organised as follows. In Section 2, the concepts of structural similarity and relevant SSIM index for image quality assessment are reviewed. Then the new proposed rough content-based image quality assessment metric is demonstrated in Section 3. In this section, the rigorous mathematical explanation of the rough fuzzy integral is provided first. After the theoretical demonstration, we illustrate the rough fuzzy integral-based metric for image quality assessment in details. In Section 4, a large amount of the experimental results are shown and we also compare the proposed approach with other methods to highlight the advantage of the proposed metric. Finally, the concluding remarks are made in Section 5.

\section{Structural similarity and SSIM index measurement}

As demonstrated in the introductory section, there exists an observation that the pixels in the natural image are highly correlated and structured, especially for the pixels spatially close to each other. Extracting such kind of structural information is the major object of the visual observation. Therefore, the measurement of the distortion of the structure information (the loss of the structure similarity) provides a good approximation to the perceived image quality. For this purpose, an SSIM index is suggested to quantify the structural similarity of each pixel sample in an image (Wang et al., 2004).

Since the structure of the object being observed is independent of the illumination and the major impact of illumination change is the variation of the average luminance and the contrast in the image, the influence of the luminance and the contrast can be separated locally from the more important structure information. Once the information of the image is categorised into three parts: luminance information, contrast information and structure information, the similarity measurement is split into three comparisons correspondingly as shown in Figure 1.

The luminance, contrast and structure comparison function are respectively defined as follows:

$$
\begin{aligned}
& l(x, y)=\left(2 \mu_{x} \mu_{y}+c_{1}\right) /\left(\mu_{x}^{2}+\mu_{y}^{2}+c_{1}\right) \\
& c(x, y)=\left(2 \sigma_{x} \sigma_{y}+c_{2}\right) /\left(\sigma_{x}^{2}+\sigma_{y}^{2}+c_{2}\right), \\
& s(x, y)=\left(\sigma_{x y}+c_{3}\right) /\left(\sigma_{x} \sigma_{y}+c_{3}\right)
\end{aligned}
$$

where $(x, y)$ represents the corresponding point pair from the original and distortion images respectively. $\mu_{x}$ is the local mean intensity at $x$, which is an estimation of the 
luminance at $x ; \delta_{x}$ is the local standard deviation at $x$, which is an estimation of the contrast at $x ; \delta_{x y}$ is the local correlation coefficient between $x$ and $y$, which is an estimation of the similarity between $x$ and $y$. The constant $c_{1}, c_{2}, c_{3}$ are included to avoid instability when the denominators are very close to zero. $c_{1}, c_{2}, c_{3}$ are small constants given by the formula

$$
\left\{\begin{array}{l}
c_{1}=\left(K_{1} L\right)^{2} \\
c_{2}=\left(K_{2} L\right)^{2} \quad K_{1}<<1, K_{2}<<1, \\
c_{3}=c_{2} / 2
\end{array}\right.
$$

where $K_{1}, K_{2}$ and $L$ are the dynamic ranges of the pixel values. The general form of the structural similarity (SSIM) index between $x$ and $y$ is defined as

$$
\operatorname{SSIM}(x, y)=[l(x, y)]^{\alpha}[c(x, y)]^{\beta}[s(x, y)]^{\gamma} .
$$

Figure 1 Diagram of the structural similarity (SSIM) measurement system (see online version for colours)

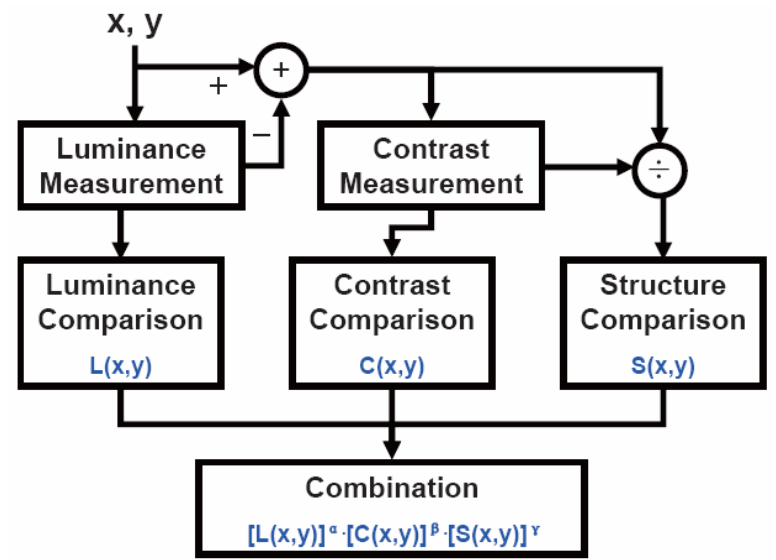

Similarity Measure

Specifically, we get $\alpha=\beta=\gamma=1$ and the resulting SSIM index is given by

$$
\operatorname{SSIM}(x, y)=\frac{\left(2 \mu_{x} \mu_{y}+c_{1}\right)\left(2 \sigma_{x y}+c_{2}\right)}{\left(\mu_{x}^{2}+\mu_{y}^{2}+c_{1}\right)\left(\sigma_{x}^{2}+\sigma_{y}^{2}+c_{2}\right)} .
$$

Once the SSIM index for each pixel of the image is calculated, the overall image quality can be measured using a weighted mean SSIM (MSSIM) index as follows:

$$
M S S I M=\sum_{j=1}^{N} W_{j} \cdot \operatorname{SSIM}_{j},
$$

where $N$ is the total number of the pixel samples and $W_{j}$ is the weight to the $j$-th pixel samples and $\sum_{j=1}^{N} W_{j}=1$. 


\section{The proposed image quality metric}

In our proposed image quality assessment method, the rough fuzzy integrals are employed into the block of pixels to quantify the image quality. To better understand this technique, the relevant concept of rough fuzzy integral is first illustrated.

\subsection{Fuzzy measure and rough fuzzy integral}

To understand the concept of rough fuzzy integral, let us start with the concept of fuzzy integral and fuzzy measures. The theory of fuzzy measures and fuzzy integrals is first introduced by Sugeno in 1972. Fuzzy measure can be considered as generalisation of the classical probability measure. A fuzzy measure $\mathrm{g}$ over a set $X$ satisfies the two conditions:

$1 g(\Phi)=0, \Phi$ is empty set.

2 if $E \subseteq F$, then $g(E) \leq g(F)$.

As a special case of fuzzy measure, the Sugeno $\lambda$-measure is defined as follows: Let $X=\left\{x_{1}, \cdots, x_{n}\right\}$ be a finite set and $\lambda \in(-1,+\infty)$. A Sugeno $\lambda$-measure is a function $\mathrm{g}$ from $2^{X}$ to $[0,1]$ with properties:

$1 \quad g(X)=1$

2 if $A \cap B=\Phi$, then $g(A \cup B)=g(A)+g(B)+\lambda g(A) g(B)$.

Based on the definition of fuzzy measure, assume that set $X$ is finite and $f: X \rightarrow[0,1]$ is the fuzzy subset of $X$. If $g$ is the fuzzy measure over $X$, then the fuzzy integral of $f$ over $A$ with respect to the fuzzy measure $g$ can be calculated

$$
f(x) \circ g=\max \left[\min \left(\min _{x \in A} f(x), g(A)\right)\right] .
$$

The fuzzy integral can be interpreted as follows. Suppose that an object is evaluated from the point of views of a set of quality factors $X$. Let $f(x)$ denote the quality evaluation on the object when quality factors are considered and let $g(\{x\})$ denote the degree of importance of this quality factor $x \in X$. It is reasonable to consider $\min _{x \in A} f(x)$ as the best-secured quality evaluation that the object provides and $g(A)$ which is called importance measure, expresses the grade of importance of this subset of quality factor. The value obtained from comparing these two quantities in terms of the 'min' operator is interpreted as the grade of agreement between real possibilities $f(x)$, and the expectation $g$. Hence, fuzzy integration is interpreted as searching for the maximal grade of agreement between the objective evidence and the expectation.

Rough fuzzy sets present roughness of fuzzy sets and are explicated in Dubois and Prade (1990). For fuzzy set $\mu$, its rough membership functions are $\mathfrak{R}^{-} \mu$ and $\mathfrak{R}^{+} \mu$, where $\mathfrak{R}$ is an indiscernibility relation on $U$, which are introduced as

$$
\left\{\begin{array}{l}
\mathfrak{R}^{-} \mu(x)=\inf \left\{\mu(y) \mid y \in[x]_{\mathfrak{R}}\right\} \\
\mathfrak{R}^{+} \mu(x)=\sup \left\{\mu(y) \mid y \in[x]_{\mathfrak{R}}\right\} .
\end{array}\right.
$$

They constitute rough approximation of $\mu(x)$. Let $F(U, \beta, g)$ be a fuzzy measure space and $\Re$ is an indiscernibility relation on $U,|U|<\infty$, the following definitions are given. 
Definition 1: The simple functions in $(U, \beta, m)$ are functions that have the form $f=\sum_{i=1}^{n} \alpha_{i} x c_{i}$ where $\alpha_{i} \neq 0, c_{i}$ is the $\beta$-measurable set and $m$ is Lebegue measure. The simple functions are Lebegue integrable in $(U, \beta, g)$, if $m\left(c_{i}\right)<\infty$ for every index $i$.

Definition 2: The simple functions in $F$ are expressed as $\mu=\sum_{i=1}^{n} \alpha_{i} x c_{i}$, where $\left\{c_{i} \mid i \leq r\right\}$ is a collection of crisp subsets of $U$ and $\alpha_{i}$ are constants in [0,1]. We have $c_{i} \in \frac{U}{\Re_{B}}$, $i=1,2, \ldots, n$, where $\mathfrak{R}_{B}$ is an indiscernibility relation derived from attribute subset $\mathrm{B}$ of A. Moreover, $\alpha_{i}$ could be explained as $M_{i}$ or $m_{i}$, which could be expressed as:

$$
\left\{\begin{array}{l}
m_{i}=\inf \left(\mu(x) \mid x \in c_{i}\right) \\
M_{i}=\sup \left(\mu(x) \mid x \in c_{i}\right)
\end{array} .\right.
$$

Theorem 1: Assumed that $\mu$ is a simple function in $F$, then it is integrable if $g\left(c_{i}\right)<1$ for every index $i$, where $\alpha_{i} \neq 0$ and $c_{i} \in U / \Re$.

Theorem 2: Let $\mu$ be a fuzzy subset of $U$, then $\Re^{-} \mu$ and $\mathfrak{R}^{+} \mu$ are simple functions in $F$ that derived from $(U, \mathfrak{R})$.

Theorem 3: Let $\mu: U \mapsto[0,1]$ be a fuzzy subset of $F$, then $\Re^{-} \mu$ and $\Re^{+} \mu$ are integrable in F.

Theorem 4: Let $\mu: U \mapsto[0,1]$ is a $F$-measurable function, then the rough upper and lower fuzzy integrals over $A \in F$ of $\mu$ with respect to $g$ are defined as

$$
\left\{\begin{array}{l}
\int_{A} \Re^{-} \mu \circ g(\cdot)=\max _{1 \leq i \leq n}\left[m_{i} \wedge g\left(A \cap H_{m_{i}}^{-}\right)\right] \\
\int_{A} \Re^{+} \mu \circ g(\cdot)=\max _{1 \leq i \leq n}\left[M_{i} \wedge g\left(A \cap H_{m_{i}}^{+}\right)\right]
\end{array},\right.
$$

where

$$
\left\{\begin{array}{l}
H_{m_{i}}^{-}=\cup_{j}\left\{c_{j} \mid \Re^{-} \mu\left(c_{j}\right) \geq m_{i}\right\} \\
H_{M_{i}}^{+}=\cup_{j}\left\{c_{j} \mid \Re^{+} \mu\left(c_{j}\right) \geq M_{i}\right\}
\end{array} .\right.
$$

\subsection{RCBM for image quality assessment}

The proposed rough-content-based metric (RCBM) is constructed on the basis of the SSIM measure and the three-component partition as shown in Figure 2. The procedure of constructing RCBM is explained as follows: First, the local structure similarity information is extracted for each pixel pair from the original and the distorted images respectively. By analysing the content of the original and distorted images, all the pixels of the given image are partitioned into three parts according to their gradient: edges, textures and flat regions. Secondly, the pixels in each of three parts are respectively classified into the $(n \times n)$ blocks. The pixels in each group are treated as the indiscernibility members, and the rough membership functions are calculated based on the SSIM index values of each pixel calculated above. Thirdly, the similarity measurement of each part is calculated by synthesising the SSIMs of all the pixels in the 
corresponding region with rough fuzzy integral. Finally, an overall image quality is evaluated with the weighting of the similarities in three regions above. The system diagram of the proposed image quality assessment system is shown in Figure 2, which consists of three basic modules: structural information extraction, region classification and the amount information fusion. It deserves mentioning that SSIM index is modified to meet the requirement of rough fuzzy integral. The structural information similarity $s(x, y) \in[-1,1]$ is redefined as $s^{\prime}(x, y)=\frac{\left|\sigma_{x y}\right|+c_{3}}{\sigma_{x} \sigma_{y}+c_{3}} \in[0,1]$. And SSIM index is redefined as

$$
\operatorname{SSIM}^{\prime}(x, y)=[l(x, y)]^{\alpha}[c(x, y)]^{\beta}\left[s^{\prime}(x, y)\right]^{\gamma}
$$

Figure 2 Diagram of the proposed image quality assessment system (see online version for colours)

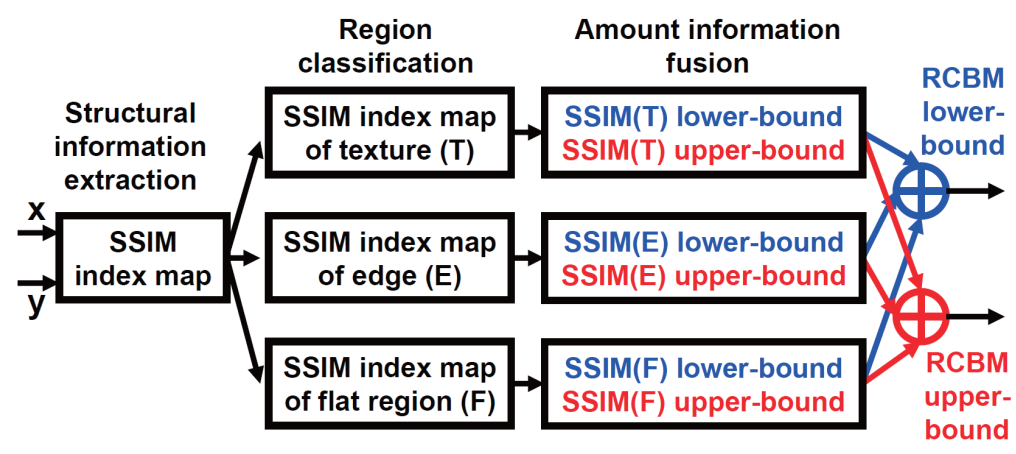

Let $X=\left\{x_{i} \mid i=1,2, \ldots, N\right\}$ and $Y=\left\{y_{i} \mid i=1,2, \ldots, N\right\}$ denote two sets, which consist of the pixels correspond to the region $A$ in the original and distorted images. Let $S=\left\{s_{i}=\operatorname{SSIM}^{\prime}\left(x_{i}, y_{i}\right) \mid i=1,2, \ldots, N\right\}$, then the overall structural information similarity of $A$ is computed as:

$$
\left\{\begin{array}{l}
\int_{A} \Re^{-}\left(\operatorname{SSIM}^{\prime}\left(x_{i}, y_{i}\right)\right) d g=\max _{1 \leq i \leq n}\left[m_{i} \wedge g\left(A \cap H_{m_{i}}^{-}\right)\right] \\
\int_{A} \mathfrak{R}^{+}\left(\operatorname{SSIM}^{\prime}\left(x_{i}, y_{i}\right)\right) d g=\max _{1 \leq i \leq n}\left[M_{i} \wedge g\left(A \cap H_{M_{i}}^{+}\right)\right]
\end{array},\right.
$$

where

$$
\left\{\begin{array}{l}
H_{m_{i}}^{-}=\cup_{j}\left\{c_{j} \mid \mathfrak{R}^{-}\left(s\left(c_{j}\right)\right) \geq m_{i}\right\} \\
H_{M_{i}}^{+}=\cup_{j}\left\{c_{j} \mid \mathfrak{R}^{+}\left(s\left(c_{j}\right)\right) \geq M_{i}\right\}
\end{array}, c_{j} \in \frac{U}{\mathfrak{R}_{B}},\right.
$$

and $\mathfrak{R}_{B}$ is an indiscernibility relation.

$$
\begin{aligned}
& \mathfrak{R}^{-}\left(s\left(c_{j}\right)\right)=\inf \left(s(p) \mid p \in c_{j}\right) \\
& \mathfrak{R}^{+}\left(s\left(c_{j}\right)\right)=\sup \left(s(p) \mid p \in c_{j}\right) \\
& m_{i}=\inf \left(s(p) \mid p \in c_{i}\right) \\
& M_{i}=\sup \left(s(p) \mid p \in c_{i}\right) .
\end{aligned}
$$


And $g(\mathrm{k})=|k| / N$, in which $|k|$ denotes the cardinality of the set $k$. The overall image quality RCBM is computed as follows:

$$
\begin{aligned}
& R C B M=\sum_{A \in\{E, T, F\}} w_{A} \cdot \operatorname{SSIM}^{\prime}(A), \\
& w_{E}+w_{T}+w_{F}=1 .
\end{aligned}
$$

\section{Experimental results}

In order to validate the proposed RCBM, we use the LIVE quality assessment database for our experiments (Sheikh et al., 2006). In the subjective database for JPEG, 29 high-resolution 24-bits/pixel RGB colour images (typically $768 \times 512$ ) were compressed using JPEG with different compression ratios to yield a database of 204 images, 29 of which were the original (uncompressed) images. The average numbers of bits per pixel compressed vary from 0.150 to 3.336. The MOS of each image is provided in the database. We take the images in Figure 3 to illustrate our experiments.

Figure 3 An illustration of the image 'Lenna', (a) original (b) distorted (c) edges (d) textures (e) flat regions

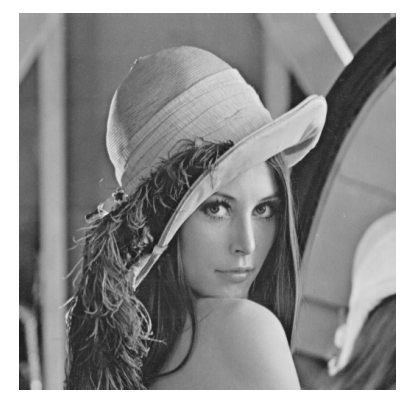

(a)

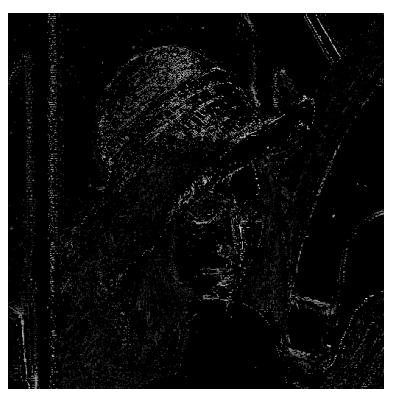

(d)

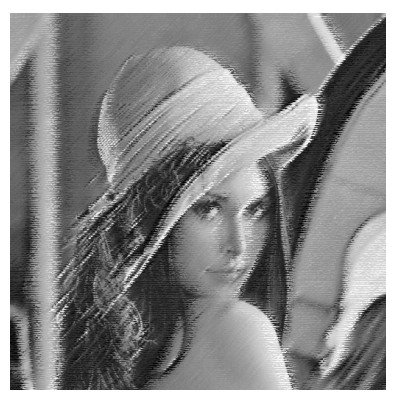

(b)

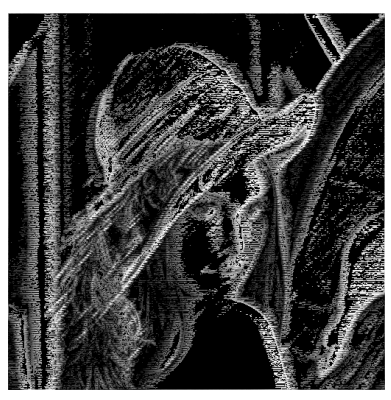

(c)

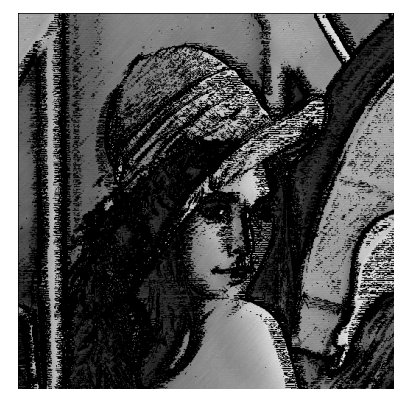

(e)

In our experiments, for simplicity we only treat the monochrome images. Since the original database is colour images, they are first transformed from RGB colour space to 
the YUV colour space and only the Y component is remained. Figure 3(a) and Figure 3(b) are the Y components of a reference colour image and a distorted JPEG image with compression ratio of $0.15771 \mathrm{bits} / \mathrm{pixel}$. Since the proposed metric requires to partition the image according to the content of the image, we also show the three regions of image 3(b) as: the edges in Figure 3(c), textures in Figure 3(d) and the flat regions in Figure 3(e).

Because the SSIM index for each pixel is directly relevant to the proposed metric, the pictorial demonstrations of the SSIM index of image $3(\mathrm{~b})$ and its three parts: the edges 3(c), the textures 3(d) and the flat regions 3(e) are shown in Figure 4, where Figure 4(a) corresponds to the values of SSIM index of the image in Figure 3(b); Figure 4(b), Figure 4(c) and Figure 4(d) are respectively represented those of edges, textures and flat regions. The relevant parameters for calculating the SSIM index above are as follows: $K_{1}=0.01, K_{2}=0.03, L=255, \alpha=1, \beta=1, \gamma=1$.

Figure 4 An example of the values of SSIM index, (a) SSIM index of the image (b) SSIM index of the edges (c) SSIM index of the textures (d) SSIM index of the flat regions

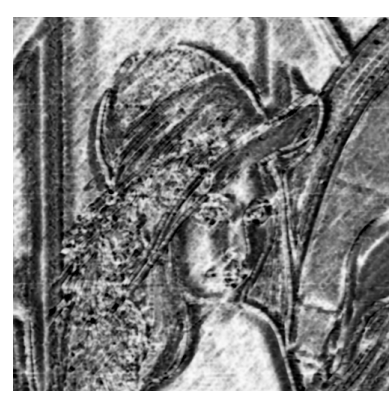

(a)

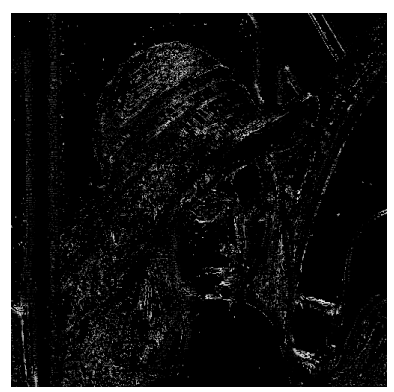

(c)

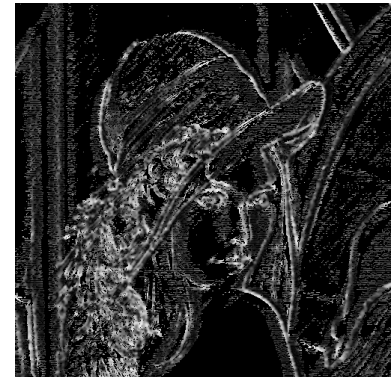

(b)

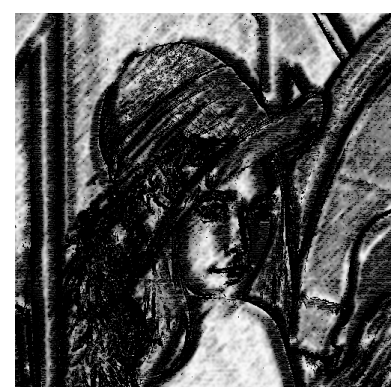

(d)

In order to get the proposed RCBM metric, the rough fuzzy integral is employed to fuse the local structural information. In this procedure, different block size can be selected for rough fuzzy integral. After the amount information fusion in each of the three regions (edges, textures and flat region), then a pair of the boundary values are obtained by weighting the three regions considering the different importance for image quality assessment. In the following experiments, we have $w_{E}=0.462, w_{T}=0.337, w_{T}=0.201$. 
Figure 5 The values of different image quality measures, (a) PSNR vs. MOS (b) MSSIM vs. MOS (c) CBM vs. MOS (d) RCBM-lower vs. MOS (e) RCBM-upper vs. MOS (see online version for colours)

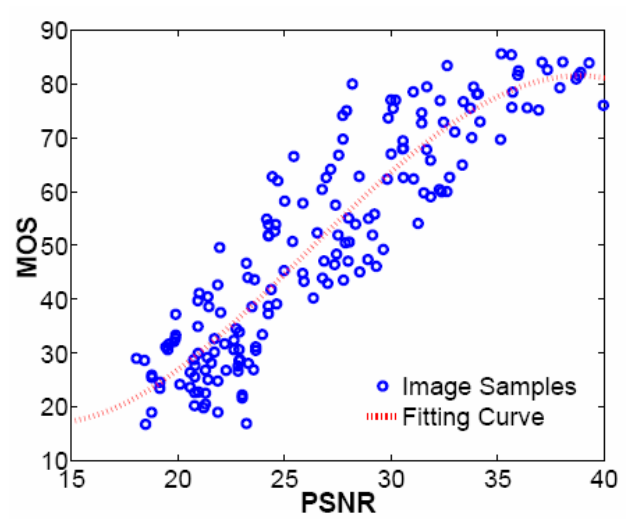

(a)

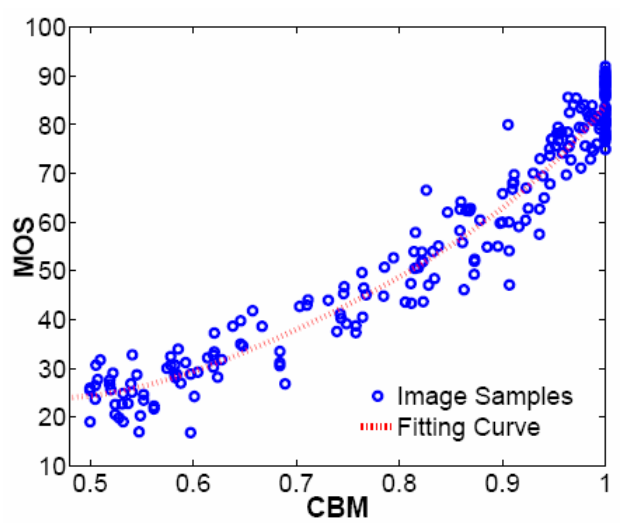

(c)

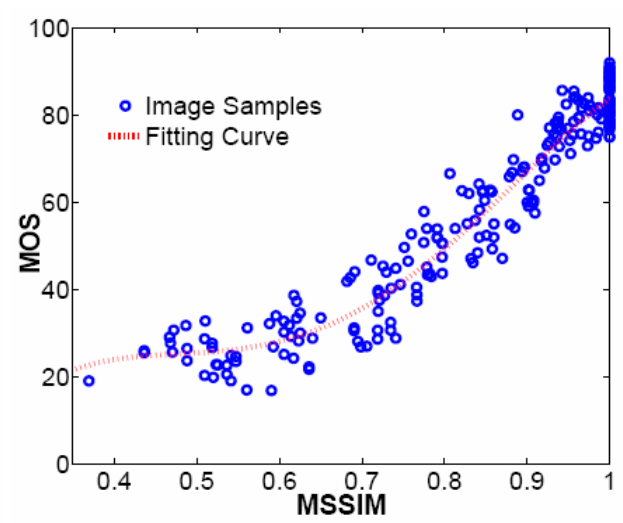

(b)

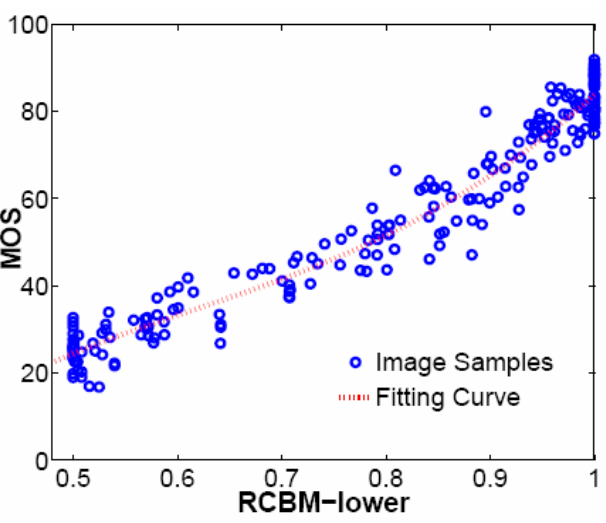

(d)

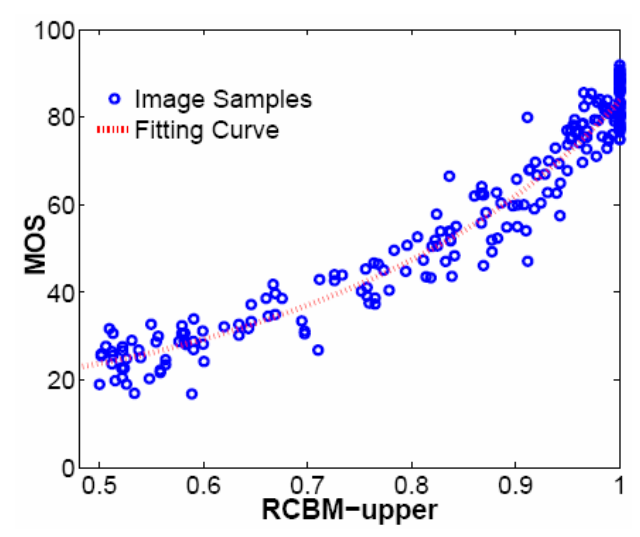

(e) 
Figure 6 Comparison of MOS-CBM and MOS-RCBM $(2 \times 2)$ (see online version for colours)

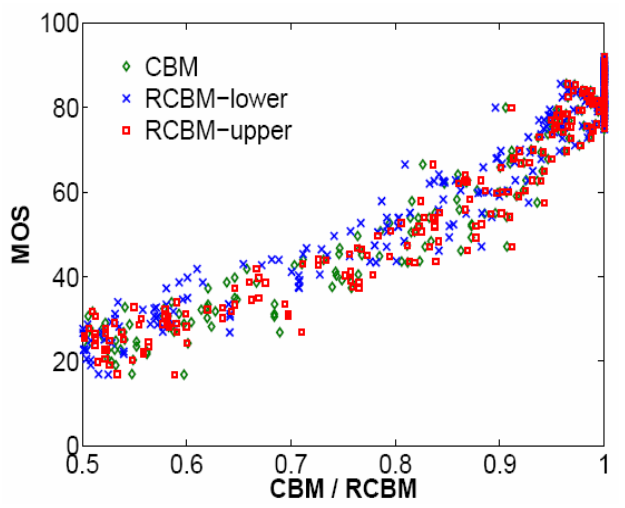

Figure 7 Comparison of MOS-CBM and MOS-RCBM $(3 \times 3)$ (see online version for colours)

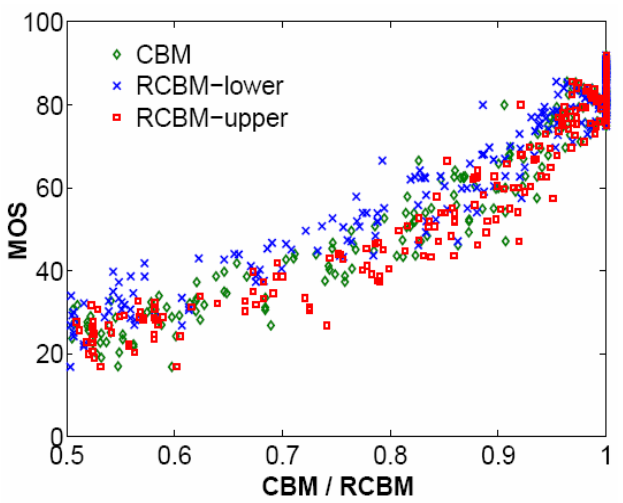

Figure 8 Comparison of MOS-CBM and MOS-RCBM $(4 \times 4)$ (see online version for colours)

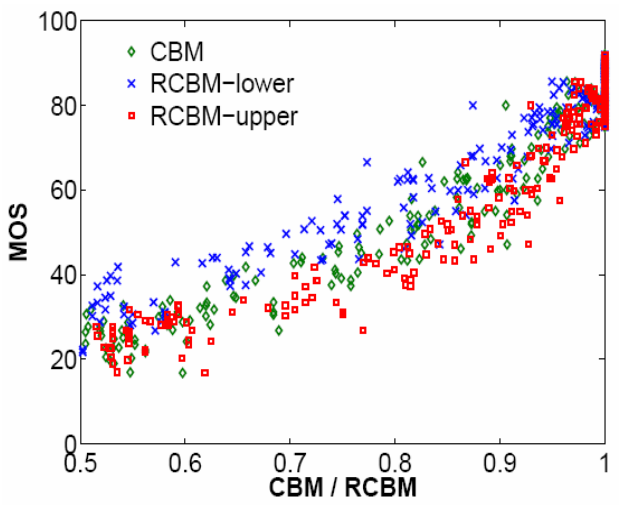

Firstly, we compare the different objective image quality metric with the subject MOSs as shown in Figure 5. Figure 5(a) to Figure 5(e) are the image quality evaluation values by using PSNR, MSSIM, CBM and RCBM respectively. In each figure, the quality 
assessment values of 233 images with different contents and distortions are shown. From Figure 5, we may observe that RCBM has the high consistency confidence versus subjective measure MOS compared with other metrics.

Next, we especially compare the CBM metric and the RCBM metric. It deserves noting that CBM can be treated as a specific instance of RCBM considering that the pixels in each of three regions are respectively classified into the $1 \times 1$ blocks (every pixel as a classification for rough fuzzy integration). In this experiment, we adopt the block sizes of $2 \times 2,3 \times 3$ and $4 \times 4$ for classification in RCBM metric, respectively. It can be seen that each CBM values are always between the RCBM lower and upper boundary values.

Table 1 Statistics of a series of 'Parrots' images

\begin{tabular}{lcccc}
\hline Images 'parrots' & Compression ratio & RCBM-lower & RCBM-upper & MOS score \\
\hline $\mathrm{a}$ & 0 (no compression) & 1.0000 & 1.0000 & 83.1968 \\
$\mathrm{~b}$ & 0.33221 & 0.9074 & 0.9242 & 60.39 \\
$\mathrm{c}$ & 0.92118 & 0.9750 & 0.9793 & 79.28 \\
$\mathrm{~d}$ & 0.31453 & 0.8997 & 0.9179 & 59.04 \\
$\mathrm{e}$ & 0.21000 & 0.7917 & 0.8327 & 47.07 \\
$\mathrm{f}$ & 0.15717 & 0.5691 & 0.5792 & 30.64 \\
\hline
\end{tabular}

Figure 9 A series of 'parrot' images, (a) image 'parrots' a (b) image 'parrots' b

(c) image 'parrots' c (d) image 'parrots' d (e) image 'parrots' e (f) image 'parrots' f

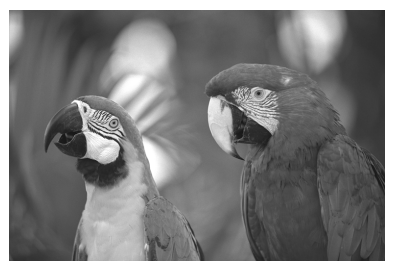

(a)

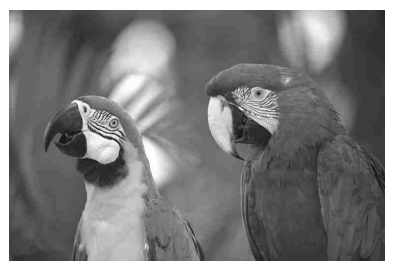

(d)

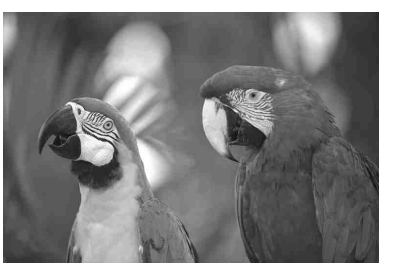

(b)

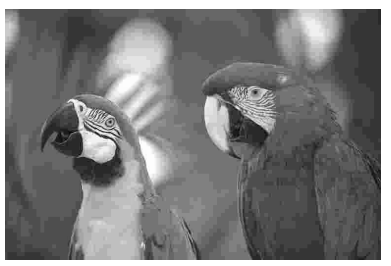

(e)

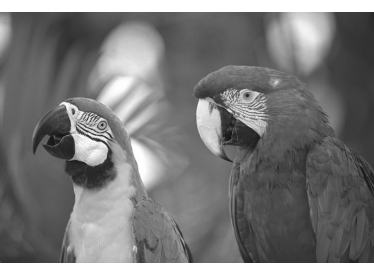

(c)

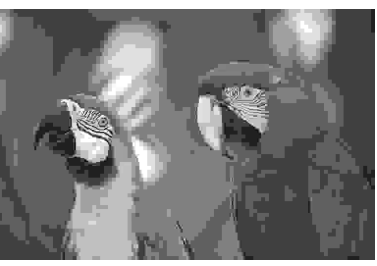

(f)

Table 2 Statistics of a series of 'house' images

\begin{tabular}{lcccc}
\hline Images 'house' & Compression ratio & RCBM-lower & RCBM-upper & MOS score \\
\hline $\mathrm{a}$ & 0.28831 & 0.6884 & 0.7333 & 36.25 \\
$\mathrm{~b}$ & 0.56641 & 0.8473 & 0.8693 & 57.85 \\
$\mathrm{c}$ & 0.32699 & 0.7292 & 0.7679 & 41.35 \\
$\mathrm{~d}$ & 1.4029 & 0.9487 & 0.9563 & 73.00 \\
$\mathrm{e}$ & 0.15798 & 0.5082 & 0.5259 & 7.77 \\
$\mathrm{f}$ & 1.8851 & 0.9678 & 0.9727 & 80.77 \\
\hline
\end{tabular}


Figure 10 A series of 'house' images, (a) image 'house' a (b) image 'house' b

(c) image 'house' c (d) image 'house' d (e) image 'house' e (f) image 'house' f

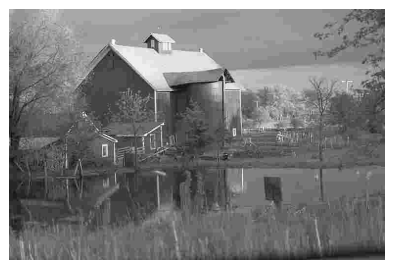

(a)

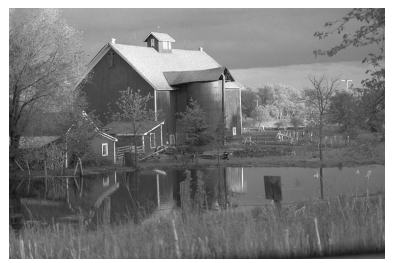

(d)

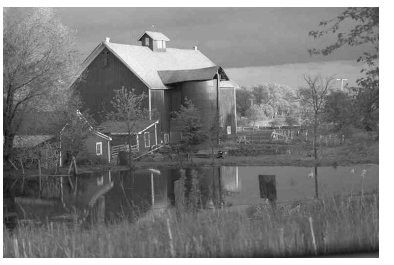

(b)

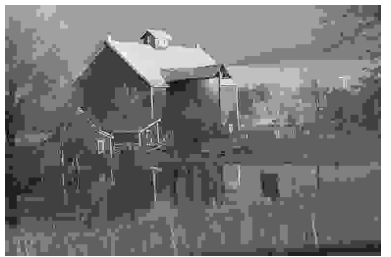

(e)

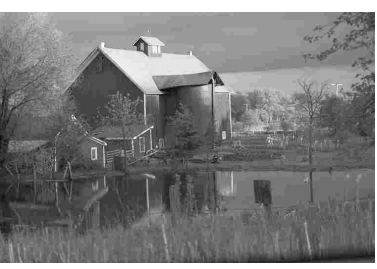

(c)

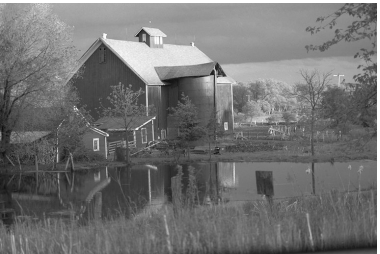

(f)

Table 3 Statistics of a series of 'monarch' images

\begin{tabular}{lcccc}
\hline Images 'monarch' & Compression ratio & RCBM-lower & RCBM-upper & MOS score \\
\hline $\mathrm{a}$ & 0.32107 & 0.8825 & 0.9116 & 39.60 \\
$\mathrm{~b}$ & 0 (no compression) & 1.0000 & 1.0000 & 75.45 \\
$\mathrm{c}$ & 0.77547 & 0.9720 & 0.9775 & 69.20 \\
$\mathrm{~d}$ & 2.6482 & 0.9937 & 0.9950 & 79.00 \\
$\mathrm{e}$ & 0.1814 & 0.6007 & 0.6689 & 25.85 \\
$\mathrm{f}$ & 0.60179 & 0.9600 & 0.9682 & 67.08 \\
\hline
\end{tabular}

Figure 11 A series of 'monarch' images, (a) image 'monarch' a (b) image 'monarch' b (c) image 'monarch' c (d) image 'monarch' d (e) image 'monarch' e (f) image 'monarch' f

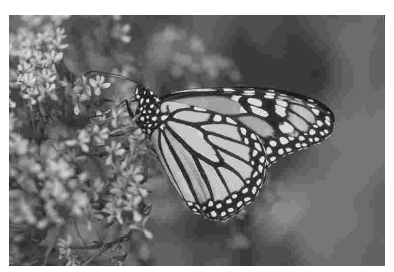

(a)

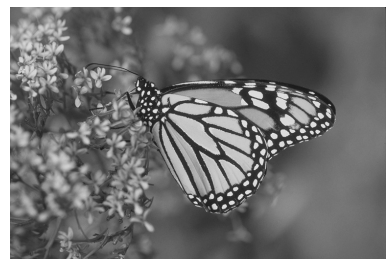

(d)

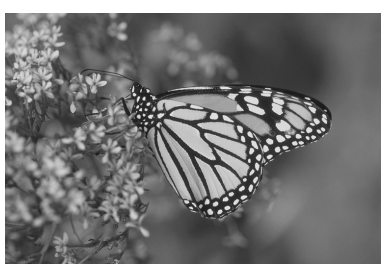

(b)

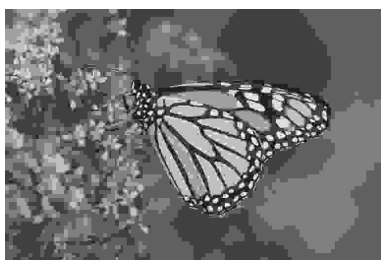

(e)

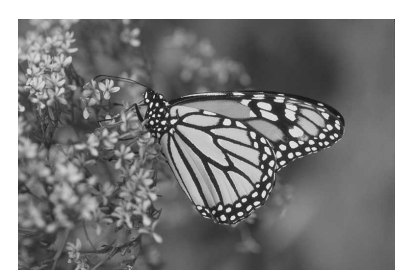

(c)

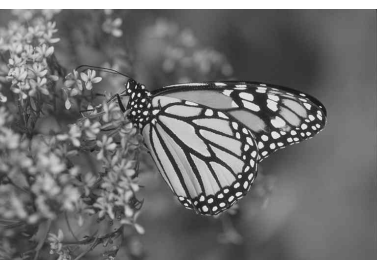

(f) 
Finally, we select three groups of the JPEG images with the different compression ratio. The quality of these images are evaluated using RCBM metric and the MOS, respectively. The experimental results are shown in Figure 9, Figure 10 and Figure 11 and listed in Table 1, Table 2 and Table 3.

From the experimental results, we can see that the RCBM lower and upper boundary values are always dropping with the increasing intensity of the compression ratio. The proposed RCBM metric is consistent with subjective image quality assessment.

\section{Conclusions}

In this paper, we have suggested a rough CBM based on the rough fuzzy integrals. According to the image content, the image is partitioned into edges, textures and flat regions corresponding to different gradient. By fusing the local structural information with the theory of rough and fuzzy set, the overall image quality is evaluated. To validate the proposed metric, the whole LIVE subjective database for JPEG is used for the experiments. We analyse and compare the RCBM with the other objective image quality assessment methods, such as PSNR, MSSIM, and CBM. The experimental result shows that our proposed metric can effectively assess the digital image quality with a pair of boundary values and provides a more flexible measure technique under the condition of the existence of uncertainty for subjective visual observation.

\section{References}

Dubois, D. and Prade, H. (1990) 'Rough fuzzy sets and fuzzy rough sets', Int. J. General Systems, Vol. 17, Nos. 2-3, pp.191-209.

Eckert, M.P. and Bradley, A.P. (1998) 'Perceptual quality metrics applied to still image compression', Signal Process., Vol. 70, No. 3, pp.177-200.

Gao, X., Wang, T. and Li, J. (2005) 'A content-based image quality metric', in RSFDGrC 2005, September, pp.231-240.

Mannos, J.L. and Sakrison, D.J. (1974) 'The effects of a visual fidelity criterion on the encoding of images', IEEE Tran. on Information Theory, July, Vol. 20, No. 4, pp.525-536.

Miyahara, M., Kotani, K. and Algazi, V. (1998) 'Objective picture quality scale (PQS) for image coding', IEEE Trans. on Communications, September, Vol. 46, No. 9, pp.1215-1226.

Pappas, T.N., Safranek, R.J. and Chen, J. (2005) 'Perceptual criteria for image quality evaluation', in A.C. Bovik (Ed.): Handbook of Image and Video Processing, 2nd ed., pp.939-959, Elsevier Academic Press, MA, USA.

Sheikh, H.R., Wang, Z., Cormack, L. and Bovik, A.C. (2006) 'Live image quality assessment database release 2' [online] http://live.ece.utexas.edu/research/quality (accessed 20 September 2011).

Sugeno, M. (1972) 'Fuzzy measure and fuzzy integral', Trans. SICE, Vol. 8, No. 2, pp.95-102.

Wang, Z. and Bovik, A. (2009) 'Mean squared error: love it or leave it? - A new look at signal fidelity measures', IEEE Signal Processing Magazine, January, Vol. 26, No. 1, pp.98-117.

Wang, Z., Bovik, A., Sheikh, H. and Simoncelli, E. (2004) 'Image quality assessment: from error visibility to structural similarity', IEEE Trans. on Image Processing, April, Vol. 13, No. 4, pp.600-612. 